\title{
Towards a cost modelling framework for outsourcing ERP systems
}

\author{
E. Shehab ${ }^{\mathrm{a}}$, M. Thomassin and M. Badawy \\ Decision Engineering Centre, Manufacturing Department, Cranfield University, \\ Cranfield, Bedford, MK43 0AL, UK.
}

\begin{abstract}
ERP systems are costly, complex and require high-qualified people to manage them. In the current economic cliamt, when companies need to cut off costs, avoid expensive in-house skilled people and being focused on the core of their businesses, outsourcing ERP systems could be a solution to consider. The aim of this paper is to provide a framework that represents the first step towards the development of a cost modelling framework for outsourcing ERP systems which may help companies to select ERP providers and understand real outsourcing costs.
\end{abstract}

Keywords. ERP systems; outsourcing; implementation; agent based modelling

\section{Introduction}

The large expansion of the Internet during the last decade is one reason for the appearance of the Application Service Providers (ASPs). Using the Internet, companies can have access to achieving IT infrastructures which can support their ERP systems. The creation of this new business model was based on three elements: allowing companies to be focused on their core businesses, reducing costs of ERP adoption and avoiding spending money on in-house expertise [1].

ERP systems have become the heart of companies' information system since the last decade. According to Payne [2], ERP systems are "an approach to the provision of business support software that enables companies to combine the computer systems of different areas of the business - production, sales, marketing, finance, human resources, etc. - and run them off a single database". The concept of the single database is the core of the system. Thus, the departments of a company can share data and communicate in an easy way. Moreover, McAdam and Galloway [3] estimate that ERP systems permit "standardising business processes, ensuring integrity of data, and removing the number, complexity, and expense surrounding old independent legacy systems".

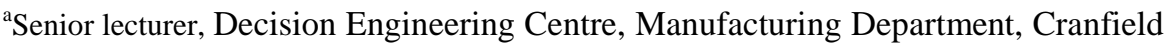
University, Cranfield, Bedford, MK43 0AL, UK. Tel: +44 (0) 1234750111 ext 5241. Fax: +44 (0) $1234754 \quad 605$; e.shehab@cranfield.ac.uk; www.cranfield.ac.uk/sas/aboutus/staff/shehabe.html 
Due to the complexity of outsourced ERP systems, many elements are at stake and make it difficult to evaluate its real cost. A framework to evaluate the real cost of outsourced ERP solutions will help companies to get a better understanding and will give them clues for decision-making in the case of adopting ERP systems.

Adoptaion of ERP outsourcing can avoid costs such as IT investments, staff recruiting and the need for in-house expertise making huge cost savings. However, outsourcing ERP systems also imply additional costs that do not exist in a more classical ERP adoption: knowledge transfer, communication systems, transition, etc. Therefore, a question arises. What is the real cost involved in the outsourcing of an ERP system? This research paper aims at understanding the main costs implicated during the outsourcing of the ERP system, especially the different activities implied during the implementation phase and at investigating how a cost model could be implemented using a modelling technique, such as agent based modelling or system dynamics.

\section{Literature Review}

Nowadays, the challenges of globalisation, with increasing competition, lead companies to find ways and means to structure their businesses so as to be flexible, reliable and meeting business demands. Typical ERP systems benefits include coordination, communication and organisational efficiency [4]. In 2008, companies have spent more than 20 billion Euros with ERP software firms [5].

However, installation of ERP systems can imply high risks, due to exceeding time and money investment. Hidden costs such as training for employees or maintenance make ERP systems installation risky. All these risks make ERP outsourcing through ASPs very interesting, as well as its lower costs and its increased flexibility.

Outsourcing is the best solution for companies which cannot afford paying for an in-house ERP adoption. The risks associated to the outsourcing are mainly about security issues; however companies avoid the risks associated to the installation and the maintenance of an in-house ERP system [6]. ERP Systems can be outsourced overseas (offshore). Even if outsourcing can save money, there is always the fact that ERP systems are too critical to be outsourced for many companies. The risks of downtime and loss of data are the biggest concerns for companies [6]. That is the reason why most of the companies which decide to outsource their ERP systems only outsource some modules which will not affect the company if a problem occurs. Outsourcing ERP systems is not only about cost, it is also a way for companies to be up-to-date concerning the latest technologies to be competitive. Thanks to outsourcing, they can have the latest software that other companies do not have yet in-house for the reason of extra cost or the need of expertise [7].

Outsourcing ERP system is complex and its implementation is quite close to a traditional ERP implementation. A contract is defined mixing customers and contractors inputs. Therefore, the final contract is composed on one hand of specification of services and of the delivery method. And on the other hand the customer defines the framework and all the legal phrases [8].

In order to understand ERP outsourcing, modelling is an essential step to get a good understanding of the AS-IS and TO-BE of the business processes. The modelling phase will avoid troubles such as lack of understanding of processes, or 
bugs and faults when the solution is implemented or tested. Besides, modelling allows optimising systems before their implementations.

Two kinds of models have been determined: the analytical model and the simulation model [9]. The analytical model depends on the number of parameters as inputs. The simulation model depends on a set of rules that will define how the model will evolve in the future according to its present state. The simulation model is commonly used when time dynamics is important. There are different approaches in simulation modelling, such as Agent Based Modelling (ABM) or System Dynamics Approach (SDA). The selection of a modelling tool is based on the level of abstraction and the way time is defined in the system that will be modelled.

The literature review has highlighted a lack of knowledge focused on outsourcing ERP systems based on modelling techniques. In order to fill this gap, a first analysis of reasons for outsourcing ERP systems will allow to get the key reasons for this new business model and then will allow to get the real cost drivers involved. Consequently, a better understanding of the cost drivers will also help to get a better understanding of the ERP activities during the implementation phase.

\section{Analysis of ERP Outsourcing}

Onshore, nearshore and offshore are three different types for outsourcing ERP systems. The classification is based on the service provider in relation to the receiving company. Companies have to benchmark the different opportunities for outsourcing their ERP system in the best way, which is extremely critical, to avoid troubles in the future that may influence their entire businesses.

According to Van Everdingen et al. [10], criteria for selecting providers who offer outsourced ERP system services are the product functionality, the implementation speed, the cost effectiveness, the interfacing with other products and the leadership in outsourced ERP system. Moreover, other criteria extracted from case studies [8] are that the scope is well defined, the duration of the engagement, the communication infrastructure, the cultural compatibility, the number of competent and skilled people available and finally the degree of implication of users needed.

ERP outsourcing has to be selected not for cost reduction only. The speed of implementation, skilled people, new businesses in other countries are some of the benefits of outsourcing that have to be seriously taken into account when companies contact ERP service providers. Then, offshore IT infrastructures can mean firing people within the company, which can be felt as a betrayal for employees. Offshore ERP systems can also create jobs (support for instance) in customers' countries. Besides, cost savings made by companies who have invested in other countries can be used to create new businesses, which means that new jobs will be created.

Culture is also a key challenge for companies who outsourced their ERP systems. In fact, customers have to match their policies and standards to meet providers' culture. If so, a comprehensive service level agreement is one tool which will avoid troubles and make the outsourcing in good conditions. Communication and implication are two of the several keys elements to successfully outsource. Here is one example extracted from a case study. A company has selected an ERP provider and decided to offshore its ERP system. Once the offshore model is in place, there is a huge loss of productivity from the 
offshore team (especially due to culture differences). The customer has to build a skilled internal team to communicate with the offshore team. All the ROI is lost in the loss of productivity and the creation of a new team internally.

Finally, companies are reluctant to use outsourcing techniques because of the risks. Data is too sensitive to be somewhere else, in another country. Companies are frightened by the fact that their data 'could' be in the middle of nowhere if the provider is not serious enough or if its security is not good enough.

\subsection{Overview of Outsourcing ERP Cost Drivers}

Identifying the cost drivers is important to get an overview of the main activities of outsourced ERP systems. Based on the literature review and real case studies [8] of companies who have outsourced their ERP systems, Figure 1 represents the nine cost drivers.

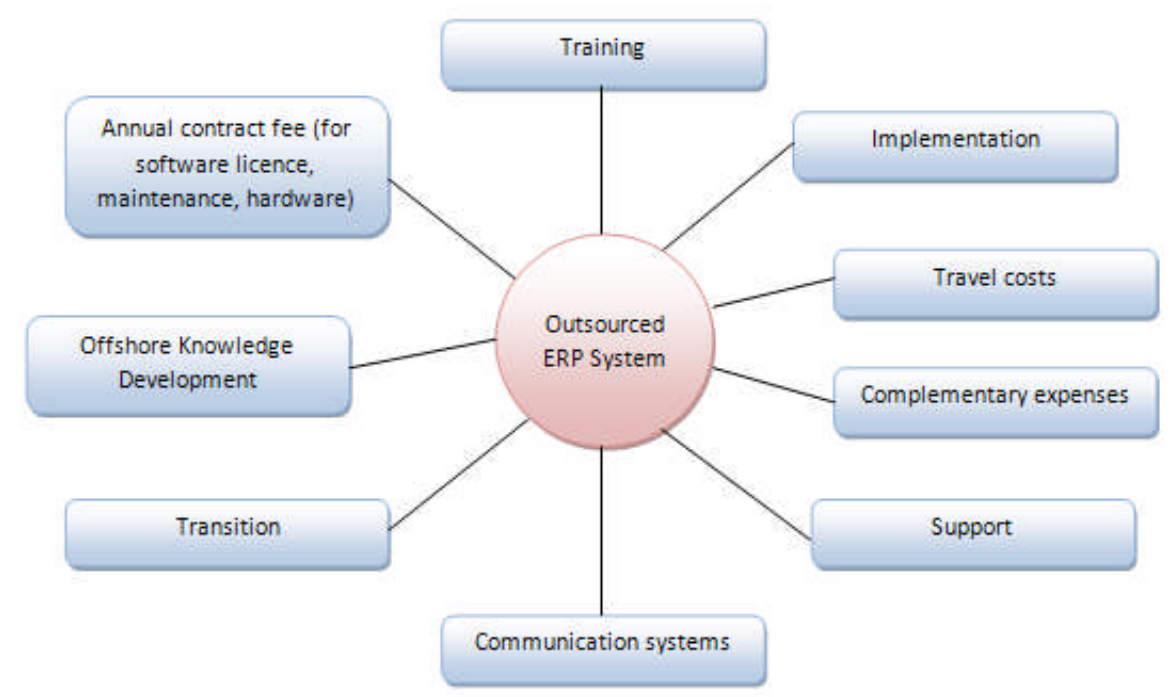

Figure 1. Cost drivers of outsourced ERP systems

\subsection{ERP implementation phases}

In order to get the real cost for an outsourced ERP adoption, it is important to know the main activities implied in the implementation phase.

An outsourced implementation phase is mainly different from a classical ERP implementation. The activities can be divided into two parts: activities which are executed onsite (it could be on the customer site directly or on a nearshore site) and activities which are executed offsite (offshore site).

The onsite team is onshore or nearshore in order to be close to the customer. Even if activities can be subdivided between the onsite and offsite teams, these two teams have to work hand in hand in order to lead the ERP adoption to success.

The onsite team aims basically at defining the customer's need (scope of the project, targets, etc.), the development of the specifications (functional, technical, processes); the testing of the system and finally the support to the users. Once the specifications done all along the ERP implementation process, they are sent to the 
offsite team in order to transform the specifications into technical developments to build up the customized ERP system. The onsite team can prepare training within the company with the end users and the offsite team can prepare specific training that can be done online. The support is done by the onsite team whereas all the problems discovered by the users on the system are solved by the offsite team.

To summarize, the onsite team aims at designing the system with the customer whereas the offsite team aims at developing the specifications coming from the onsite team into a system. Figure 2 illustrates the activities involved in each team through the ERP implementation phases.

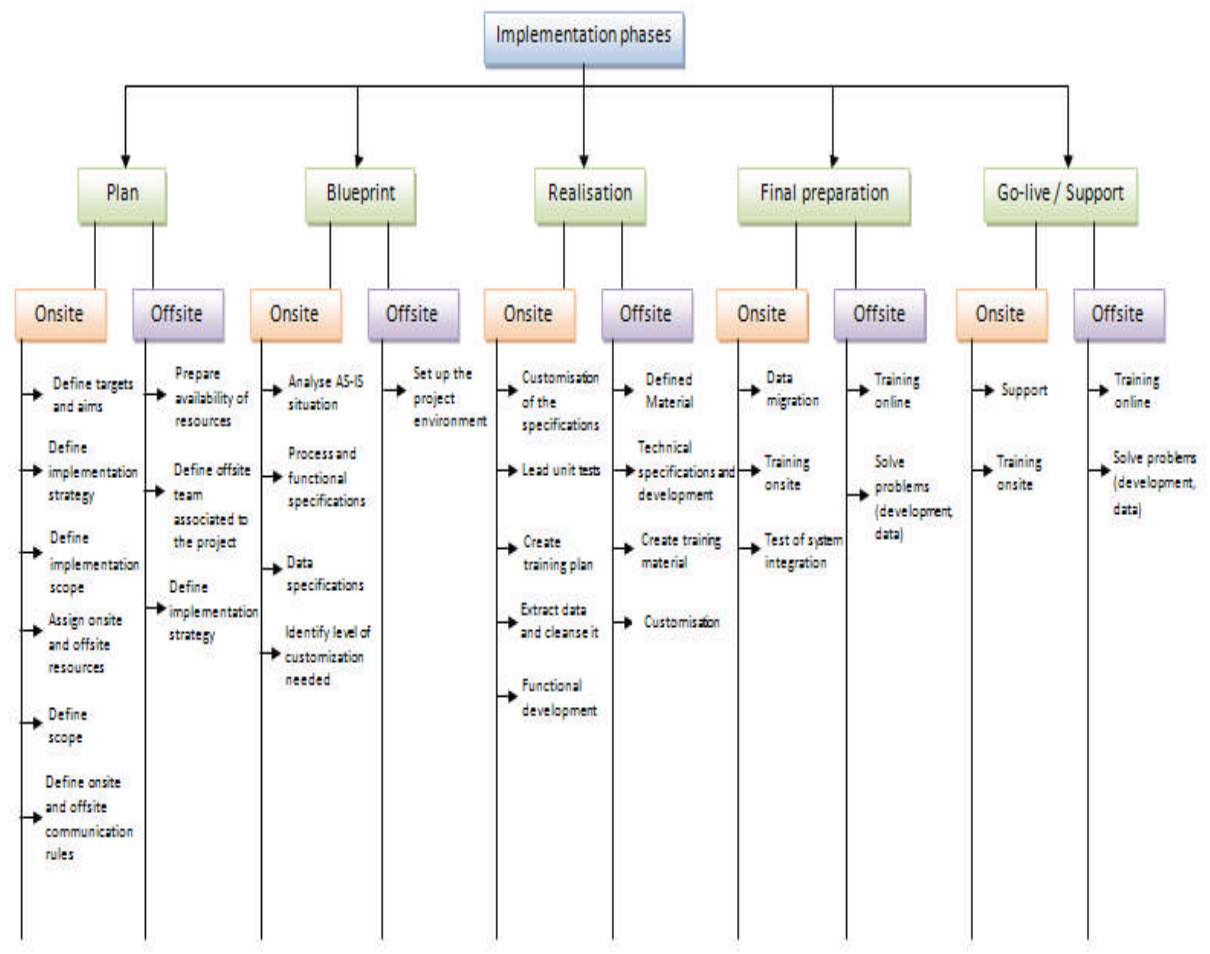

Figure 2. ERP Implementation Activities

\section{Agent Based Modelling Analysis}

An agent based modelling analysis has been employed to model the cost drivers and the activities of the ERP implementation. Agent based modelling technique requires [11] to: (i) Identify agents and their behaviour; (ii) Identify agents relationships; (iii) the location of the provider; (iv) Associate data to agents; (v) Validate the agent behaviour models and (vi) Run the model and analyse the outputs. It is common to use UML for representing models as object-oriented. Modelling agents is the first step to analyse a real world situation such as outsourcing ERP systems. It allows understanding the relationships between the agents and their parameters. 


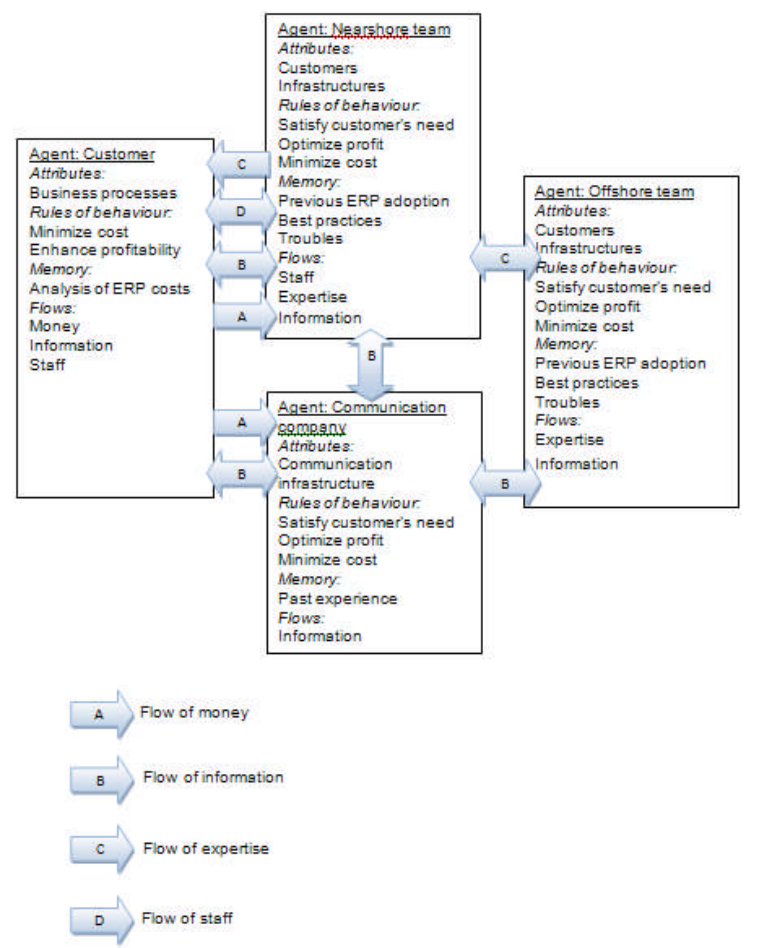

Figure 3. Agents in outsourcing ERP systems

Figure 3 illustrates the interactions between the four major agents in outsourcing ERP systems. It shows the case of a customer who wants to outsource offshore using a nearshore team which will be the link between the customer and the offshore team. Then the customer does not communicate directly with the offshore team, but with the nearshore team which relays the needs of the customer to the offshore team. At this level, four types of elements are exchanged between agents: money, information, expertise and staff. Money will be used to get the cost of outsourcing. Information is composed of the communication (such as needs from the customer), expertise is knowledge coming from the provider (nearshore and offshore teams) which will allow to develop the ERP system (implementation, etc.). Finally, the staffs are considered as a flow because of the training period which involves people to move (users to the nearshore team or vice versa).

Attributes are the main elements that agents own. For instance, customers have business processes, the provider has infrastructures and the communication company has communication infrastructure. Rules of behaviour are rules that will conduct agents' behaviours. For instance, all the agents have a rule which recommends minimizing costs. Finally, agents have to rely on their memory if they are facing a situation that had already happened in the past.

Figure 4 shows the environment in which agents will evolve and will affect their actions and interactions. The environment conditions the manner agents will interact. Agents in the same environment will be able to communicate easily. However, two agents in two different bubbles will have to use the communication tool (which will be the communication company agent) in order to be able to communicate. Nevertheless, if an agent from the customer wants to communicate and interact with an agent of the offshore team environment, this agent will not be 
able to communicate directly with it. The agent will have to go through the nearshore team environment and interact with a nearshore team agent that will relieve the request to the offshore team. This environment model is the closest to the reality (customers negotiate with the nearshore team and only the nearshore team has contacts with the offshore team).

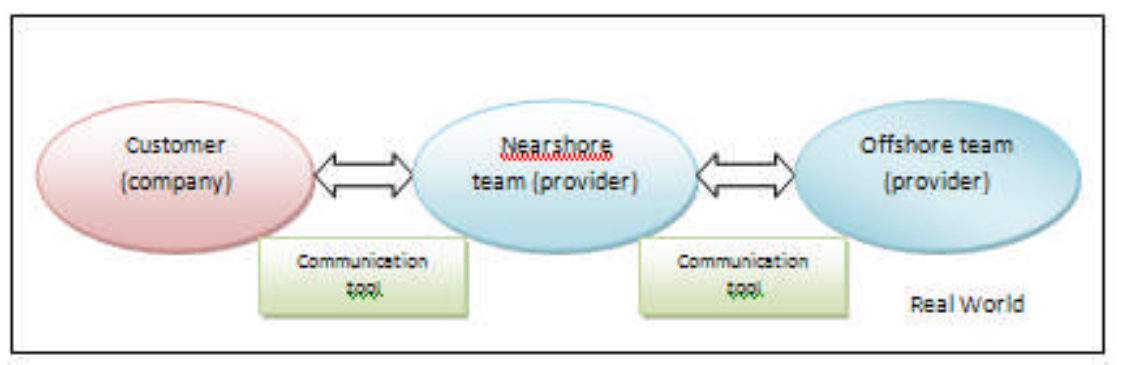

Figure 4. Environment for Agents

Based on the ERP implementation activities diagram (Figure 2), the UML class diagram illustrated in Figure 5 represents a general overview of agents, methods and attributes.

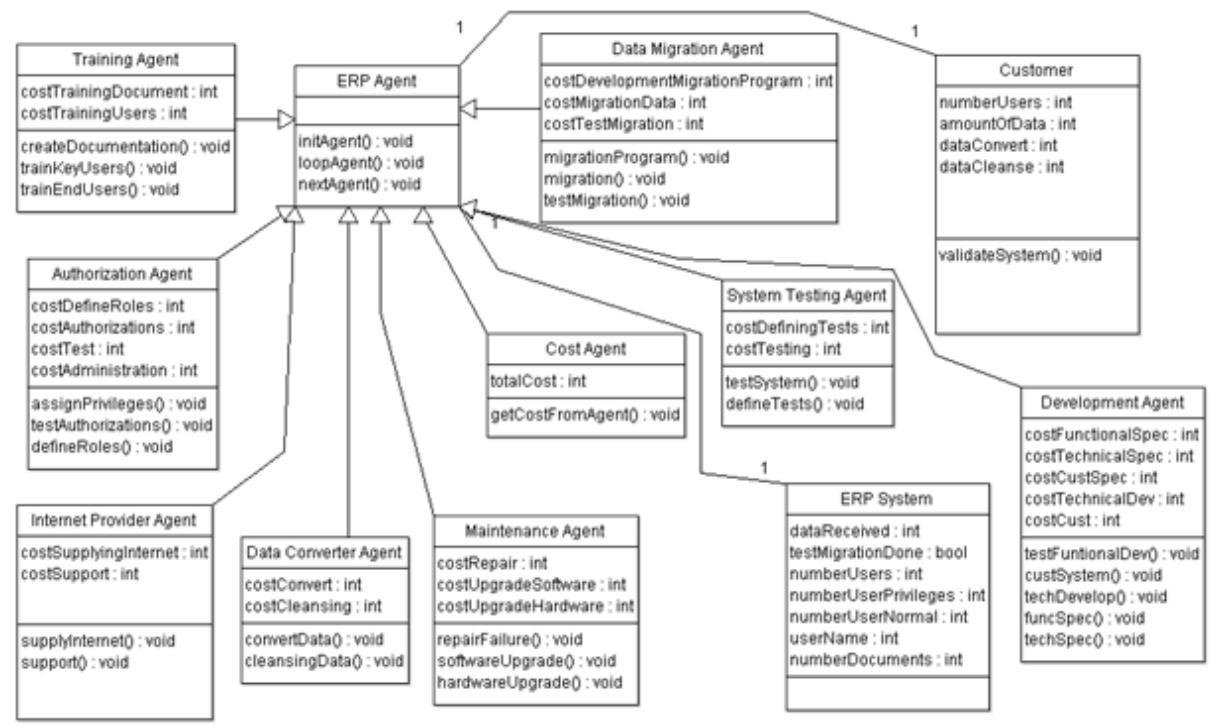

Figure 5. Implementation UML Class Diagram

\section{Conclusions}

The paper presents a framework which will assist in developing cost modelling of outsourcing ERP. ERP Cost Drivers and ERP implementation activities have been developed. An Agent Based Modelling analysis and UML have been created. Outsourcing ERP systems will be given a fully comprehensive literature review to 
surround the problem. Moreover, the costs involved are explained, as well as the activities for the implementation. In the case people would like to know the real cost of outsourcing, the model is presented and ready to be implemented and customized according to the need. Finally, outsourcing is a real alternative to classical in-house ERP adoption. The selection of the provider is crucial and can lead to a successful ERP implementation or to a total reverse with huge money losses.

\section{REFERENCES}

[1] Ekanayaka Y, Currie WL, and Seltsikas P. Delivering enterprise resource planning systems through application service provider. Logistics Information Management 2002; 15(3); 193-203.

[2] Payne W. The time for ERP?. Work Study 2002; 51 (2); 91-93.

[3] McAdam R. and Galloway A. Enterprise resource planning and organisational innovation: a management perspective. Industrial Management \& Data Systems. 2005; 105 (3); 280-290.

[4] Stevens C. Enterprise resource planning: a trio of resources. Information Systems Management. 2003; 20 (4); 61-67.

[5] Shehab EM, Sharp MW, Supramaniam L and Spedding TA. (2004) "Enterprise resource planning: An Integrative Review. Business Process Management Journal. 2004; 10 (4); 359-386.

[6] Newell M. Leveraging opportunities for an ERP implementation to driver enterprise transformation. An Everest White Group Whitepaper. 2008.

[7] Olson DL. Evaluation of ERP outsourcing. Computers \& Operations Research. 2007; 34 (12); 3715-3724.

[8] ERPwire (2009), 'Is ERP outsourcing necessary for all organizations?', available: http://www.erpwire.com/erp-articles/erp-outsourcing-necessity.htm (Accessed on August 1,2009).

[9] Hendel A, Messner W and Thun F. (2008), 'Rightshore! Successfully Industrialize SAP Projects Offshore', Springer.

[10] Borshchev A and Fillipov A. From system dynamics and discrete event to practical agent based modeling: Reasons, techniques, tools. Proceedings of the 22nd International Conference of the System Dynamics Society, July 25-29, 2004.

[11] Van Everdingen Y, van Hillegersberg and Waarts E. ERP adoption by European midsize companies. Communications of the ACM. 2000; 43 (4); 27-31.

[12] Macal C. and North M. Tutorial on agent-based modelling and simulation part 2: how to model with agents. Proceedings of the 2006 Winter Simulation Conference, Karlsruhe, Germany; 2006; 73-82. 